\title{
Hautpflege bei Psoriasis
}

\author{
Eine klinisch-experimentelle, multizentrische \\ Untersuchung
}

\begin{abstract}
Zusammenfassung. Eine speziell zur Reinigung und Pflege der psoriatischen Haut entwickelte Produktserie ${ }^{1}$ wurde in experimentellen Untersuchungen und im Anwendertest auf ihre Eigenschaften geprüft. Dabei zeigten die Reinigungs- und Pflegeprodukte eine Verbesserung verschiedener Hautparameter (Erhöhung der Feuchtigkeit, Verminderung der Rauigkeit, Reduzierung der Schuppen, Reduzierung des Juckreizes) bei Stabilisierung der Hautbarrierefunktion. Der Pilz Malassezia furfur, ein möglicher Triggerfaktor für Kopfschuppen, wurde durch das Shampoo in seiner Quantität deutlich reduziert. Die positiven $\mathrm{Er}$ gebnisse der experimentellen Studien $(n=60)$ konnten zusätzlich in zwei Anwendertests an 177 bzw. 50 Psoriatikern bestätigt werden. Die Pflegeserie erfuhr eine hohe kosmetische Akzeptanz und kann für die tägliche Anwendung auch im Sinne einer Prophylaxe empfohlen werden.
\end{abstract}

Skin Care in Psoriasis. The efficacy and safety of a new developed range of care products ${ }^{1}$ especially for psoriatic skin has been tested in experimental studies and user trials. Both cleansing and care products showed significant improvement of different skin parameters like moisture, roughness, scales or itch. The amount of Malassezia furfur on the scalp was significantly reduced. The positive results of the experimental studies $(n=60)$ were confirmed by usertrials with 177 and 50 people suffering from psoriasis. The complete range was cosmetically well accepted and can be recommended for daily care.

\section{Einleitung}

Psoriatiker mit chronisch-stationärer Form sollten neben der Anwendung spezifischer Therapeutika eine therapiebegleitende, schonende, konsequente Hautpflege betreiben; dies gilt besonders auch für das erscheinungsfreie Intervall [3].

Dabei ist die Zufuhr rückfettender und feucht haltender Bestandteile zur Erhaltung bzw. Regeneration der Hautbarrierefunktion [11] ebenso wichtig wie eine Linderung des häufig empfundenen Juckreizes $[1,5,7]$. Es ist vorteilhaft, wenn die
J. Barth', H.-P. Nissen², W.-G. Ocker ${ }^{3}$, H. Prieur ${ }^{4}$

${ }^{1}$ Hautarztpraxis, Borna

2 DermaConsult, Alfter

${ }^{3}$ Hermal, Reinbek

${ }^{4}$ Hautarztpraxis, Duisburg
Grundlage dem jeweiligen Hauttyp angepasst ist. Die Waschzubereitungen sollten mild reinigen und rückfetten, während das Shampoo die Schuppen reduzieren und die Haare und Kopfhaut pflegen sollte.

In diesem Bemühen wurde von der Firma Hermal eine Pflegeserie $^{1}$, bestehend aus Wash Lotion, Shower Gel, Shampoo, Hand \& Body Cream (H \& B Cream) und Body Lotion (internationale Produktnomenklatur), in Zusammenarbeit mit Dermatologen und Betroffenen entwickelt. Diese Pflegeserie wurde mit bewährten hautphysiologischen, biochemischen bzw. mikrobiologischen Methoden geprüft und in einer breiten Anwenderstudie getestet. Die Produkte sind frei von Parfümund Farbstoffen; die Wash Lotion, das Shower Gel und die Body Lotion enthalten zudem keine Konservierungsstoffe [8].

In die Wasch- und Pflegeprodukte sind Harnstoff (Urea) und Polidocanol inkorporiert, das Shampoo beinhaltet Climbazol, Polidocanol und Dexpanthenol.

\section{Patienten und Methoden \\ Experimentelle Untersuchungen}

Die experimentellen Untersuchungen wurden an 60 Psoriatikern (29 Männer, 31 Frauen, Altersbereich 22 - 59 Jahre) mit Plaque-Typ-Psoriasis vom Forschungsinstitut DermaConsult $\mathrm{GmbH}$ in der Zeit von November 1999 bis Juli 2000 durchgeführt.

Folgende hautphysiologische, biochemische bzw. mikrobiologische Untersuchungsmethoden kamen nach täglicher Applikation der Produkte auf die Haut über maximal 28 Tage zur Anwendung:

\section{- Transepidermaler Wasserverlust (TEWL)}

Der transepidermale Wasserverlust wurde mit Hilfe des Tewameters TM 210 (Courage und Khazaka, Köln) gemessen. Jeder Wert wurde entsprechend den Standards der Europäischen Kontaktdermatitis-Gruppe dreifach bestimmt und als Mittelwert verwendet. 
- Messung der Hautfeuchtigkeit

Das Corneometer CM 825 (Courage und Khazaka, Köln) registrierte die elektrische Kapazität der Hautoberfläche in künstlichen Einheiten. Es wurden jeweils 3 Messungen des Hautareals durchgeführt; der Mittelwert war das Maß für die Hautfeuchte des Stratum corneum.

- Messung der Rauigkeit

Die Bestimmung der Rauigkeit erfolgte mit dem Skin Visiometer (Courage und Khazaka, Köln). Eine computerunterstützte CCD-Kamera machte dabei die Berechnung von Höhenunterschieden im Hautrelief mit Hilfe der speziell entwickelten Software skin visiometer SV500 möglich.

- Bestimmung der Konzentration von Hautoberflächenlipiden (Squalen)

Die quantitative Analyse wurde mittels HPLC nach Applikation von $1 \mathrm{ml}$ Isopropanol auf die Haut unter Standardbedingungen vorgenommen. Verwendet wurde: LiChrospher ${ }^{\circledR} 100$ RP-18 Merck-Deutschland, UV 217 nm, 1 ml/ min, Beckman System Gold ${ }^{\circledR}$ (USA).

- Messung der Sebumkonzentration

Die Messungen wurden mit dem Sebumeter SM 810 (Courage und Khazaka, Köln) nach einer photometrischen Methode durchgeführt. Ein Computer berechnete den mittleren Sebumgehalt auf der Oberfläche des Messareals in $\mu \mathrm{g}$ Sebum $/ \mathrm{cm}^{2}$ Haut.

- Nachweis der Schuppenreduktion

Der Nachweis der Schuppenreduktion erfolgte gravimetrisch (mg) und subjektiv mit Hilfe eines 6-stufigen Scores. Zusätzlich wurde mit einer speziellen Nahfeld-UV-Kamera (Courage und Khazaka, Köln) eine Photodokumentation der Schuppenreduktion zur Unterstützung der gravimetrischen Daten durchgeführt.

- Nachweis der Reduktion von Malassezia furfur (M. furfur) Die oberen Schichten des Stratum corneum wurden mit selbstklebender Folie (D-Squame) gesammelt. Das Material wurde mit einer wässerigen Lösung $(0,5 \mu \mathrm{g} / \mathrm{ml})$ von Fluoreszenzfarbstoff (Sigma-Aldrich F-3397) zur Detektion von Pilzen angefärbt. Mit Hilfe der Fluoreszenzmikroskopie wurde die Anzahl pro $\mathrm{mm}^{2}$ bestimmt.

\section{Anwenderstudien}

Die Pflegeserie wurde von 177 Patienten mit Plaque-Typ-Psoriasis (96 Männer, 81 Frauen) über ca. 4 Wochen getestet. Die Produkte sollten entsprechend dem individuellen Pflegeverhalten angewendet werden, d.h. mindestens einmal täglich. Die Altersstruktur der Probanden war wie folgt: $5 \%$ unter 20 Jahre, $13 \%$ unter 30 Jahre, $26 \%$ unter 40 Jahre, $20 \%$ unter 50 Jahre, $36 \% 50$ Jahre und älter.

Die Patienten wurden gebeten, einen Fragebogen auszufüllen, in dem sie neben ihren persönlichen Daten ihren Hauttyp (fettig, normal, trocken, sehr trocken), die Lokalisation ihrer Hautveränderungen, den subjektiv empfundenen Schweregrad der Erkrankung (leicht, mittel, schwer) und die derzeit laufende Therapie eintragen sollten. Zusätzlich erfolgte eine Beurteilung der Eigenschaften bzw. Wirkungen der Produkte wie Reinigungsvermögen, Pflegeeffekte und Verträglichkeit. Die Bewertung wurde nach einem Punktescore von 1-6 (sehr gut bis ungenügend) vorgenommen.
50 weitere Patienten (je 25 Männer bzw. Frauen) füllten einen entsprechenden Fragebogen zu den Effekten des Shampoos auf Fülle, Glanz, Halt und Kämmbarkeit der Haare aus. Das Shampoo wurde ebenfalls über einen Zeitraum von etwa 4 Wochen angewandt.

\section{Biometrie und Statistik}

Alle Daten wurden zentral erfasst und mit PC-Standardsoftware für statistische Kenngrößen (n, Min, Max, Mittel, Median, etc.) ausgewertet. Das 5\%-Niveau wurde als Grenze der statistischen Signifikanz angenommen. Verwendet wurden jeweils die Daten des Prä-/Postvergleiches.

\section{Ergebnisse}

\section{Experimentelle Untersuchungen}

Transepidermaler Wasserverlust (TEWL)

Die wiederholte Bestimmung des transepidermalen Wasserverlusts als Parameter für die Hautbarrierefunktion zeigte für die Waschzubereitungen (Wash Lotion, Shower Gel) keine Veränderung, die Hautbarriere blieb auch nach wiederholter Anwendung über 2-4 Wochen intakt. Bei der Anwendung der Pflegeprodukte (Hand \& Body Cream, Body Lotion) verbesserte sich die Hautbarriere signifikant $(p<0,05)$ - abzulesen an den abnehmenden Werten des TEWL (Abb. 1).

\section{Hautfeuchtigkeit}

Die corneometrischen Messungen belegten eine leichte Erhöhung im Feuchtigkeitsgehalt schon nach Anwendung der Waschzubereitungen; nach Anwendung der Pflegeprodukte H\&B Cream und Body Lotion stieg die Hautfeuchte um 21\% bzw. $28 \%$ an (Abb. 2). Für alle Produkte war die Verbesserung der Hydratation der Hornschicht statistisch signifikant $(\mathrm{p}<0,05)$.

Entsprechend den gemessenen Daten zur Hautfeuchtigkeit (Abb. 2), brachte auch die subjektive Beurteilung der Verbesserung der Hauttrockenheit bei 54\% der Anwender gute bis sehr gute Ergebnisse (vgl. Tab.1).

Tab. 1 Bewertung der Wasch- und Pflegeprodukte $(N=177)$

\begin{tabular}{lllll}
\hline & $\begin{array}{l}\text { Gesamt- } \\
\text { urteil }\end{array}$ & positiv & neutral & negativ \\
\hline Hautempfindung* $^{*}$ & 2,3 & $67 \%$ & $28 \%$ & $3 \%$ \\
Hautglätte/-weichheit* & 2,4 & $63 \%$ & $30 \%$ & $3 \%$ \\
$\begin{array}{l}\text { Produktverträglichkeit* } \\
\text { weniger }\end{array}$ & 2,0 & $82 \%$ & $12 \%$ & $5 \%$ \\
$\begin{array}{l}\text { Hautaustrocknung* } \\
\text { Reinigungseffekt* }\end{array}$ & 2,5 & $54 \%$ & $38 \%$ & $5 \%$ \\
\hline
\end{tabular}

\footnotetext{
* fehlende Prozente $=$ keine Angabe
} 
Hautrauigkeit

In Korrelation zur Hautfeuchtigkeit wurden auch die messbare und die empfundene Hautrauigkeit nach Anwendung der Pflegeprodukte statistisch signifikant $(p<0,05)$ geringer (Abb. 3 ). Nach 4-wöchiger Anwendung der Pflegeprodukte hatte sich die Hautglätte um über $20 \%$ verbessert, die Haut zeigte ein einheitlicheres Hautrelief.

Im Anwendungstest gaben 63\% der Probanden eine gute bis sehr gute Hautglätte bzw. Hautweichheit schon nach $2-4$ Wochen (vgl. Tab.1) an.

Konzentration von Sebum und Hautoberflächenlipiden (Squalen)

Für beide Waschzubereitungen wurde die Sebum- bzw. Squalenkonzentration über die Zeit bestimmt. Während die Werte beim Duschgel über die 14 Tage Untersuchungszeit praktisch unverändert blieben, zeigte sich vor allem bei der Sebumkonzentration für die Wash Lotion ein leichter Anstieg (o. Abb.). Insgesamt wiesen alle Messungen für die beiden Waschprodukte eine gute Rückfettung der Haut aus; eine entfettende Wirkung wurde in keinem Fall gemessen.

\section{Schuppenreduktion}

Sowohl die objektive gravimetrische Messung als auch das subjektive Empfinden der Probanden zeigte, dass nach Anwendung des Shampoos eine deutliche, statistisch signifikante $(\mathrm{p}<0,05)$ Schuppenreduktion stattgefunden hat (Abb. 4).

Die subjektive Einschätzung ergab eine Reduktion der Schuppenmenge nach 28 Tagen von 52\%. Der Rückgang der Schuppenbildung wurde gravimetrisch mit $45 \%$ Reduktion objektiviert und durch Aufnahmen mit einer speziellen UV-NahfeldFotodokumentation bestätigt (Abb. 5).

\section{Reduktion von Malassezia furfur}

Der Pilz M. furfur ( = Pityrosporum ovale) wurde durch die eingesetzte Konzentration von 0,5\% Climbazol schnell und wirkungsvoll zurückgedrängt. Die Daten zeigten schon nach wenigen Anwendungen eine statistisch signifikante $(p<0,05) A b-$ nahme (Abb. 6).

\section{Sicherheit und Verträglichkeit}

Bei den dermatologisch-experimentellen Untersuchungen wurden keine Unverträglichkeitsreaktionen oder Irritationen gefunden. Eine Sicherheitsbewertung gemäß Kosmetikverordnung $§ 5 b$ liegt für alle Produkte vor [8].

\section{Anwenderstudien}

Die Angaben der Probanden zum Hauttyp wiesen mit $51 \%$ bzw. $20 \%$ einen trockenen bzw. sehr trockenen Hauttyp aus, 25\% empfanden ihren Hauttyp als normal und nur 4\% stuften ihre Haut als fettig ein. Bezüglich der Lokalisation der PsoriasisHerde wiesen bei möglichen Mehrfachnennungen 78\% einen Befall der Extremitäten, 55\% einen Befall des Kopf-/Halsbereiches und $51 \%$ einen Befall des Rumpfes aus. 30\% beurteilten



Abb. 1 TEWL-Messungen über die Zeit.

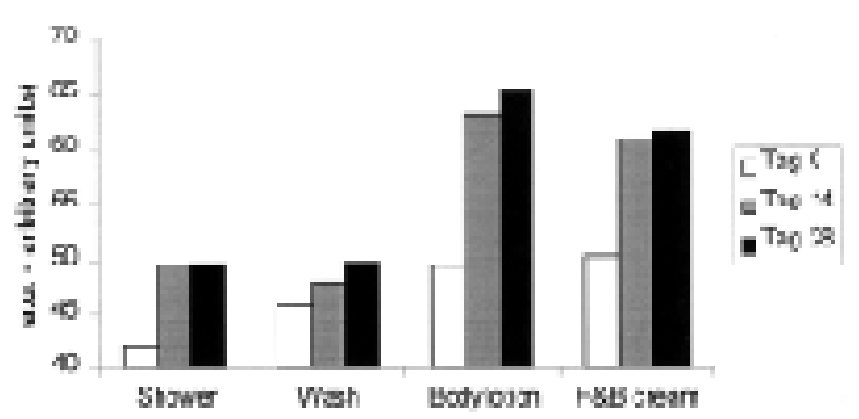

Abb. 2 Hautfeuchtigkeitsbestimmung über die Zeit.

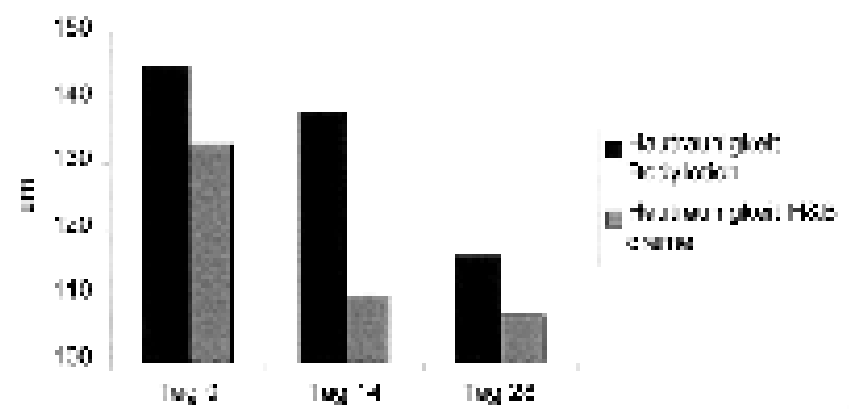

Abb. 3 Hautrauigkeit

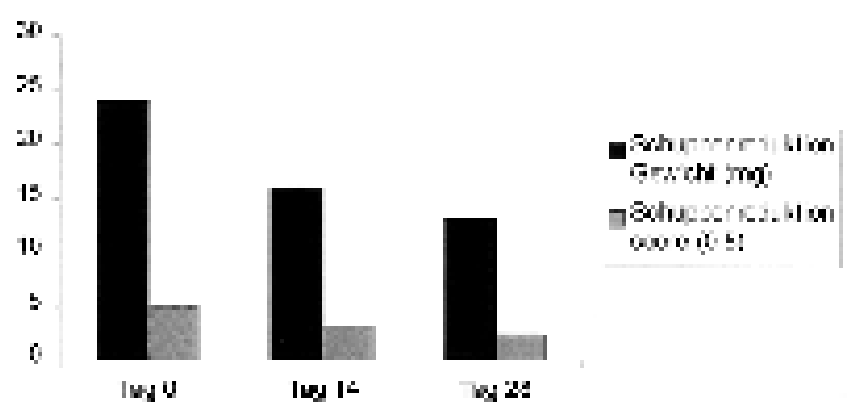

Abb. 4 Reduktion der Schuppen unter Shampoo-Anwendung. 
a

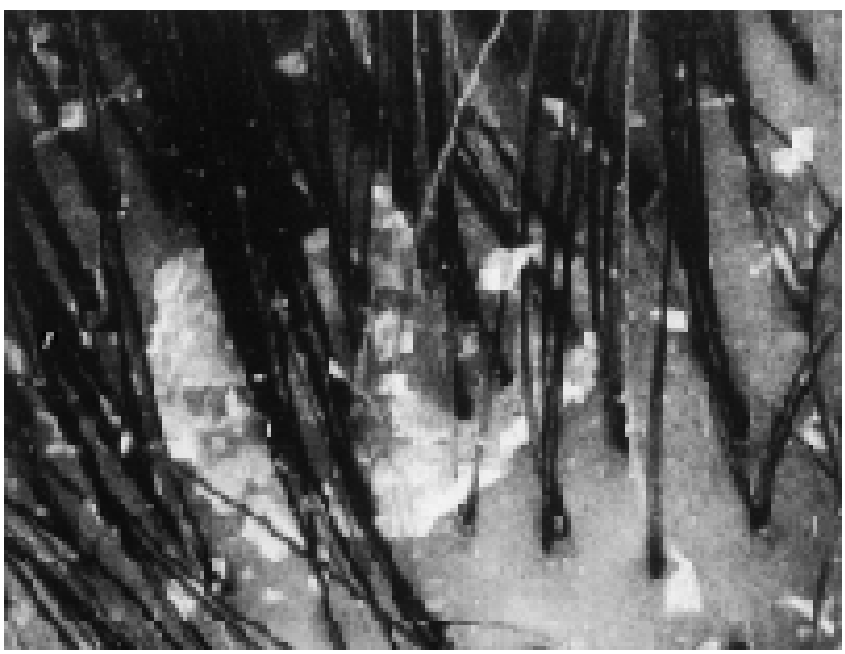

b

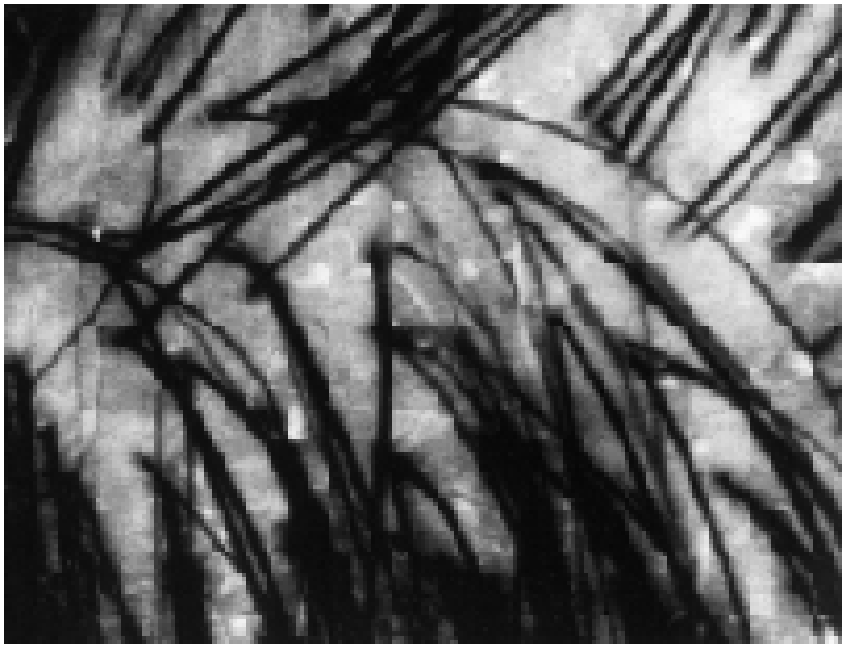

Abb.5 UV-Nahfeld-Fotodokumentation, Prä/Post-Darstellung der Schuppenreduktion.

den Schweregrad ihrer Krankheit als leicht, $48 \%$ als mittelschwer und 20\% als schwer. Der Rest machte keine Angaben.

\section{Bestimmung der Juckreizlinderung}

Die Patienten des Anwendungstests wurden mit Hilfe eines Fragebogens befragt, ob ein vorhandener Juckreiz gelindert werden konnte. Insgesamt gaben $83 \%$ der Befragten an, Juckreiz zu empfinden. Für alle Produkte konnte eine gute bis sehr gute Juckreizlinderung durch das Lokalanästhetikum Polidocanol (Laureth 6.5 bzw. Lauromacrogol) bestätigt werden. Bei den Pflegeprodukten war diese mit 92 - 100\% häufiger als bei den nur kurzfristig auf der Haut verbleibenden Waschzubereitungen mit etwa $70 \%$. Das Shampoo zeigte bei 86\% der Anwender eine juckreizlindernde Wirkung (o. Abb.).

\section{Bewertung der Eigenschaften durch Patienten}

Die subjektiven Angaben der Patienten zu den Eigenschaften/ Effekten der Wasch- und Pflegeprodukte sind in Tab. 2 zusammengefasst.

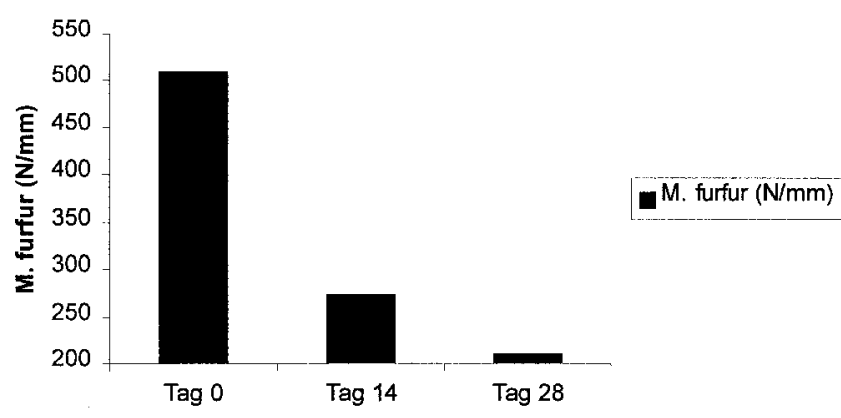

Abb. 6 Bestimmung von M. furfur.

Sowohl die Produktverträglichkeit als auch die Produkteffekte an der Haut beurteilten die Probanden überwiegend positiv. Nur in wenigen Einzelfällen wurden kurzfristige, lokale Irritationen beobachtet, die dem für Harnstoff beschriebenen Stinging-Effekt zugeschrieben wurden.

Die positive Bewertung der Pflegeserie konnte auch für das Shampoo bestätigt werden, wie Tab. 2 ausweist.

Zudem konnte gezeigt werden, dass eine zeitgleiche Anwendung von Therapeutika und Pflegeprodukten ohne subjektive Beeinträchtigung möglich ist. 27\% $(n=48)$ der Patienten berichteten über eine gleichzeitig erfolgte Lokaltherapie, vor allem mit steroidhaltigen Zubereitungen (12\%) und Vitamin$\mathrm{D}_{3}$-Analoga (8\%), aber auch mit Teer und Dithranol.

\section{Diskussion}

Die sowohl von Patienten als auch von Dermatologen erhobenen Forderungen nach einer Pflegeserie eigens für die psoriatische Haut wird mit der Produktserie erfüllt. Dabei werden die besonderen Anforderungen an derartige adjuvante Pflegepräparate berücksichtigt: Es wurden wenig irritierende, die Hautfeuchtigkeit erhöhende, die Rauigkeit vermindernde, Schuppen und Juckreiz reduzierende Wirkstoffe verwendet, die die Hautbarriere schützen und den Lipidfilm der Hautoberfläche intakt halten oder regenerieren $[7,8,11]$. Dies rechtfertigt zugleich ihren Einsatz als Basistherapeutika.

Die Umsetzung dieser Forderungen an die Pflegeprodukte konnte durch hautphysiologische bzw. biochemische Untersuchungen belegt werden [8]. Hinzu kommt eine signifikante Reduzierung des anthropophilen Pilzes M. furfur durch das im Shampoo enthaltene Climbazol [6]. Für M. furfur wird eine Triggerfunktion im pathogenetischen Geschehen der Psoriasis diskutiert $[1,9,10]$.

Harnstoff als natürlicher Feuchtigkeitsfaktor in der Haut hat sich zur Therapie und Pflege seit langem bewährt [11] und trägt wesentlich zu den positiven Pflegeeigenschaften der Produktserie bei. Das eingesetzte Lokalanästhetikum Polidocanol wirkt auf die sensiblen Nervenfasern, reduziert den eventuell vorhandenen Juckreiz $[2,5]$ und vermindert somit die Gefahr eines Köbner-Phänomens [10]. In einer aktuellen Untersuchung [7] wurden bei einer breiten Patientenbefragung als wesentliche Beeinträchtigungsfaktoren durch Psoriasis Symptome wie Juckreiz, Schuppung, Sichtbarkeit, Erythem und tro- 
Tab. 2 Bewertung des Shampoos $(\mathrm{N}=50)$

\begin{tabular}{lllll}
\hline & $\begin{array}{l}\text { Gesamt- } \\
\text { urteil }\end{array}$ & positiv & neutral & negativ \\
\hline Haarglätte & 2,1 & $70 \%$ & $26 \%$ & $4 \%$ \\
Verträglichkeit & 1,6 & $84 \%$ & $16 \%$ & $0 \%$ \\
Glanz & 2,4 & $60 \%$ & $32 \%$ & $8 \%$ \\
Fülle & 2,3 & $50 \%$ & $48 \%$ & $2 \%$ \\
Kämmbarkeit & 2,2 & $66 \%$ & $26 \%$ & $8 \%$ \\
Reinigung & 2,3 & $78 \%$ & $22 \%$ & $0 \%$ \\
Schuppenreduktion & 2,4 & $58 \%$ & $42 \%$ & $0 \%$ \\
Empfinden & 2,1 & $68 \%$ & $30 \%$ & $2 \%$
\end{tabular}

ckene bzw. empfindliche Haut identifiziert. Bei einer adjuvanten Pflegeserie, die diesen Beeinträchtigungen entgegenwirkt, ist eine gute Compliance zu erwarten.

Die aus den experimentellen Untersuchungen abgeleiteten Erwartungen wurden im Anwendertest bestätigt [8]. Die Produktverträglichkeit wurde als gut bis sehr gut eingestuft und die überwiegende Mehrheit der Patienten registrierte eine Verbesserung des Hautzustandes. Die kosmetische Akzeptanz der Produkte und die Compliance der Anwender waren generell gut bis sehr gut.

Eine zeitgleiche Anwendung der Pflegeprodukte mit antipsoriatisch wirkenden Therapeutika kann nach vorliegenden Untersuchungen [8] als unbedenklich betrachtet werden.

Die Reinigungs- und Pflegeserie kann zur wirksamen regelmäBigen Pflege der erscheinungsfreien oder nur wenig befallenen psoriatischen Haut auch im Sinne einer Prophylaxe empfohlen werden.

\section{Danksagung}

Wir danken den beteiligten Ärzten bzw. Institutionen:

H.-J. Böhmer, Hautarztpraxis/Unna; Deutscher Psoriasis Bund/ Hamburg; E.-W. Jecht, Hautarztpraxis/Nürnberg; G. A. Lutz, Hautarztpraxis/Köln; U. Mrowietz, Universitätshautklinik/ Kiel; H.W. Niedecken, Hautarztpraxis/Rheinbach

\section{Literatur}

${ }^{1}$ Baker BS, Powles A, Garoich JJ, Hardman C, Fry L. Differential Tcell reactivity to the round and oval forms of Pityrosporum in the skin of patients with psoriasis. Brit J Dermatol 1977; 136: 319-325

2 Bernhard JD. Itch mechanisms and management of pruritus. Health profession division, chapter 3. McGraw-Hill, 1994

${ }^{3}$ Finlay AY. Emollients as adjuvants therapy for psoriasis. J Dermatol Treatment 1977; 8: 25-27

${ }^{4}$ Fluhr JW, Gehring W. Messung der Hornschichtfeuchtigkeit mit unterschiedlichen elektrophysikalischen, nicht-invasiven Bioengineering-Verfahren. Akt Dermatol 2000; 26: 171-173

${ }^{5}$ Gupta MA, Gupta AK, Kirkby S, Weiner HK, Mace TM, Schork NJ, Johnson EH, Ellis CN, Voorhees JJ. Pruritus in psoriasis. Arch Dermatol 1998; 124: $1052-1057$

${ }^{6}$ Schmidt A, Rühl-Hörster B. In vitro suspectibility of Malassezia furfur against azole compounds. Mycoses 1996; 39: 309-312

${ }^{7}$ van de Kerkhof PCM, de Hoop D, de Korte J, Cobelens SA. Patient compliance and disease management in the treatment of psoriasis in The Netherlands. Dermatology 2000; 200: 292-298

${ }^{8}$ Kosmetikdossier/Product Information package: Hermal, data on file. 2000

${ }^{9}$ Meinhof W. Pityrosporum-Hefen als Krankheitserreger. Akt Dermatol 1995; 21: $366-370$

${ }^{10}$ Rosenberg EW, Belew PW. Improvement of psoriasis of the scalp with ketoconazole. Arch Dermatol 1982; 118: 370-371

${ }^{11}$ Schweig T. Reinigen, Pflegen, Stabilisieren - Hilfen für die trockene Haut. Pharm Z 1998; 143 (43): 11 - 16

Prof. Dr. med. J. Barth

Hautarzt/Allergologe Rudolf-Virchow-Straße 4 04552 Borna 